\title{
Una alternativa para la producción de superficies de doble curvatura en láminas de fibrocemento a través de mecanizado de tres ejes
}

\section{An alternative to produce doubly curved surfaces in fiber cement sheets using three-axe machining}

\author{
Ramiro Isaza-Escobar a, Camilo Andrés Páramo-Velásquez ${ }^{\text {b }}$, Felipe Oviedo-Sierra ${ }^{\mathrm{c}}$ \\ \& Hugo Ripoll de la Barrera ${ }^{d}$ \\ a Gestor de Proyectos, Red Tecnoparque SENA Nodo Medellín - Grupo GIGAT, Servicio Nacional de Aprendizaje SENA, \\ Medellín-Colombia, risaza@sena.edu.co \\ ${ }^{b}$ Gestor de Proyectos, Red Tecnoparque SENA Nodo Medellín - Grupo GIGAT, Servicio Nacional de Aprendizaje SENA, \\ Medellín-Colombia. cparamov@sena.edu.co \\ ${ }^{c}$ Gestor de Proyectos, Red Tecnoparque SENA Nodo Medellín - Grupo GIGAT, Servicio Nacional de Aprendizaje SENA, \\ Medellín-Colombia, felipeoviedo@misena.edu.co \\ ${ }^{c}$ Líder SENNOVA - Centro para el Desarrollo del Hábitat y la Construcción, Grupo de Investigación Red de construcción, \\ infraestructura y servicios públicos, Servicio Nacional de Aprendizaje SENA, Medellín-Colombia, hripoll@sena.edu.co
}

Recibido: noviembre 9, 2019. Aceptado: diciembre 15, 2019.

\section{Resumen}

El presente trabajo describe un proceso alternativo de mecanizado de fibrocemento. El mecanizado se realizó en una lámina comercial de $10 \mathrm{~mm}$ de espesor, a partir de una superficie de doble curvatura en un centro de mecanizado ROLAND - MDX 40A, por medio de un proceso de desbaste y posterior acabado en 3 ejes, con el uso de herramientas de corte comerciales de carburo de tungsteno. Para la obtención de la geometría, se utilizó Rhinoceros 5 con el complemento Grasshopper para la parametrización del modelo y RhinoCAM para la generación de los códigos G que permitieron ejecutar los procesos de mecanizado. La geometría parametrizada en la lámina partió de un módulo de un paraboloide Hiperbólico, el cual tomaba sus dimensiones, según las medidas de la placa en su largo, ancho y profundidad y el número de veces que el usuario deseaba replicar en un patrón rectangular este módulo. El proceso de mecanizado se realizó sin refrigeración y con herramientas de corte estándar, por lo que la velocidad de avance, profundidad de corte y avance de la herramienta se ajustaron a parámetros mínimos que permitieron conservar la vida útil de la herramienta y el cuidado del equipo durante los procesos de mecanizado y así obtener un acabado superficial aceptable, esto tuvo como consecuencia, un tiempo lento los procesos, los cuales podrían ser optimizados utilizando sistemas de refrigeración o herramientas especializadas en el mecanizado de materiales cerámicos. La técnica de manufactura empleada en este material y desarrollada en este trabajo, puede ser aplicada en la fabricación de piezas con volúmenes bajos de producción o elementos especializados, en sectores tales como la Ingeniería y manufactura, el sector Eléctrico, la industria de la construcción y arquitectura, el arte y diseño y la fabricación de Mobiliario.

Palabras Clave: CAM; Fibrocemento; Fresado; parametrización CAD.

\begin{abstract}
The present work describes an alternative process for the machining of fiber cement. This machining was carried out on a commercial sheet, having a $10 \mathrm{~mm}$ thickness and based on a double curvature surface in a ROLAND - MDX 40A machining center. This machining was performed through a roughing and subsequent finishing process in 3 axes, using some commercial tungsten carbide cutting tools. Rhinoceros 5 was used and complemented with Grasshopper, in order to obtain the geometry and the parameterization of the model. RhinoCAM was used to generate the $G$ codes that allowed the machining processes execution. The geometry parameterized in the fiber cement sheet started from a Hyperbolic paraboloid module, with dimensions taken according to the plate measurements in length, width, and depth, and the number of times the user wanted to replicate this module in a rectangular pattern. The machining process was carried out without any refrigeration process, besides some standard cutting tools. Therefore, the main machining parameters as the speed, the cut-depth, and the tool advance were adjusted to the minimum set-points. This configuration allowed the preservation of the tool life and the care of the equipment during the machining processes. However, these parameters resulted in slow processing, which could have been optimized using cooling systems or machining tools of specialized ceramic materials. The manufacturing technique used in this material and developed in this work can be applied to the low volume production of parts in fiber cement sheets. In this way, there could be applications in the Engineering and manufacturing sector parts, to the Electric sector, the construction and architecture industry, art and design, and furniture manufacturing.
\end{abstract}

Keywords: CAM; Fiber Cement; milling; hyperbolic paraboloid; CAD parameterization.

\section{Citar como:}

R. Izaza, C. Páramo, F. Oviedo \& H. Ripoll. “Una alternativa para la producción de superficies de doble curvatura en láminas de fibrocemento a través de mecanizado de tres ejes". Revista CINTEX, Vol. 24(1), pp. 51-63. 2019. 


\section{INTRODUCCIÓN}

El fibrocemento en un material compuesto, de matriz de cemento reforzado con sílice y fibras de celulosa [1]. La industria de la construcción es el sector que tradicionalmente demanda este tipo de material en formatos de lámina que varían en su espesor y textura para la instalación de cerramientos exteriores, entrepisos y cielos rasos [2]. Este material, no solo es utilizado por sus propiedades mecánicas, térmicas y acústicas, sino también por el interés de reducir y controlar las fuentes de contaminación de este sector con materiales ecológicos [3] [4] o el aprovechamiento de residuos industriales que generen nuevas alternativas de uso [5] .

La aplicación de materiales amigables con el ambiente que no requieren de la explotación de recursos no renovables o el uso de recursos fósiles para su obtención ha implicado el uso de materiales más responsables con el ambiente [10]. El fibrocemento, al ser un material compuesto de matriz Cerámica (cemento) y refuerzo de fibras naturales (Celulosa) y sílice, presenta características de alta resistencia, tenacidad, resistencia a la fatiga, resistencia al agrietamiento y durabilidad. Por otro lado, por su Matriz Cerámica, el fibrocemento es un aislante térmico, acústico y resistente al agua a condiciones ambientales [11]. La industria de la construcción es el sector que demanda este tipo de material y que ha tenido beneficios por este, ya que es una alternativa segura, durable, responsable con el ambiente y con mejores propiedades mecánicas y químicas, en comparación a materiales tradicionales como la madera o materiales con agregados de asbesto, ya prohibido por legislación en algunos países [12]. Láminas o placas (lisas o texturizadas), son los formatos disponibles en el mercado para aplicaciones como; entrepisos, cerramientos exteriores e interiores, cielo rasos y fabricación de mobiliario [1].

El mecanizado CNC (Control numérico computarizado) de materiales tradicionales ha sido empleado especialmente por la industria metalmecánica donde la demanda de estos servicios hace que el sector aplique tecnología de punta con el fin de buscar la reducción de tiempos y mejora de la calidad para cumplir con las características técnicas demandadas por el sector industrial [6]. Por ello, explorar este tipo de tecnologías en materiales donde no se suelen aplicar estos procesos y que presentan un crecimiento en su uso, toma importancia, ya que reduce el desconocimiento del comportamiento de estos al ser mecanizados [7] [8]; tal es el caso de este trabajo, donde se exploran alternativas de mecanizado de 3 ejes para superficies de doble curvatura en láminas de fibrocemento.

Esta exploración permite trascender el uso actual que se tiene de este material y busca encontrar alternativas diferentes de aplicación en otros sectores, donde se requiera de un material con propiedades aislantes, con resistencia a las diferentes condiciones climáticas y buen comportamiento mecánico. La sección 2 presenta las características del fibrocemento como material e insumo principal, junto con las características de los equipos y herramientas utilizados; también se describen los parámetros de configuración para el mecanizado ejecutado.

\section{MATERIALES Y MÉTODOS}

\subsection{El fibrocemento}

El fibrocemento o CFRC por sus siglas en inglés (Cellulose Fiber Reinforced Cements. FRC en su forma abreviada), es un material compuesto por una matriz de cemento, reforzado con sílice, fibras de celulosa y aditivos para su conformado y estabilización química y mecánica [2] [9]. El método general de fabricación de este material es el proceso Hatschek, nombrado así por el autor de la patente registrada en 1900, y tal como se muestra en la figura 1. Este es un proceso semicontinuo de tres etapas, como se describe a continuación.

Primero, en la etapa de conformado de láminas, se transporta por cinta la mezcla de cemento y fibra aun sin solidificar al rodillo de formación de tableros. Esta cinta transportadora es alimentada por una serie de rodillos impregnados de la mezcla tomada desde un tanque de agitación continua. El uso de vacío en esta etapa reduce en gran parte el agua de la mezcla y permite la formación de una capa de $1 \mathrm{~mm}$ aproximadamente de espesor. Para la etapa de formación de tableros, la lámina formada en la etapa anterior es enrollada en un cilindro de gran tamaño, en capas sucesivas hasta alcanzar el calibre especificado. Una guillotina corta el material enrollado en el cilindro y lo desenrolla en una cinta trasportadora, para ser comprimido y moldeado por una prensa en la forma deseada. La última etapa de curado es realizada en una autoclave en condiciones de presión, aire o vapor que permitan terminar de endurecer y extraer el aire del material obtenido. [10] 


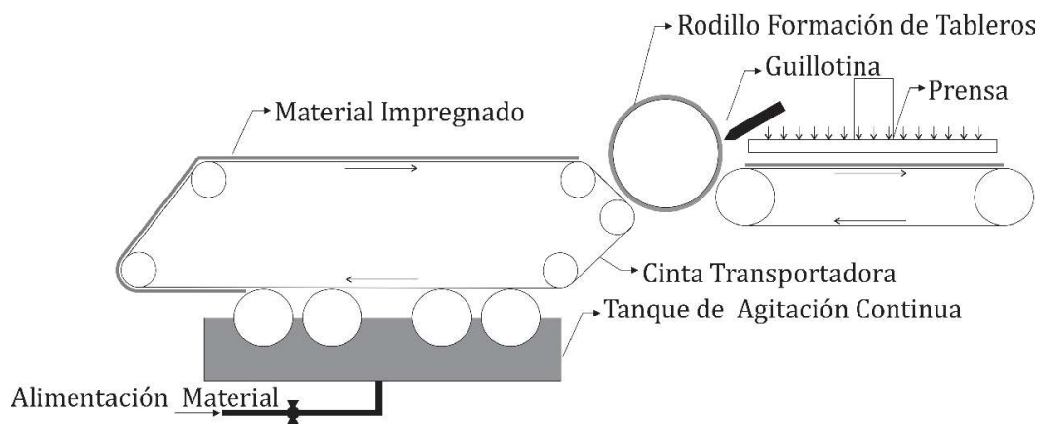

Figura 1. Método de fabricación láminas de fibrocemento por el método proceso Hatschek [3].

Las láminas de fibrocemento usualmente se comercializan en formatos de $2440 \times 1220 \mathrm{~mm}$, en espesores de $4,6,8,10,14,17,20 \mathrm{~mm}$ y pueden variar en la textura de su superficie, imitando texturas de madera, ladrillos, baldosas y otros. En particular, el fibrocemento utilizado en este trabajo es fabricado bajo la norma NTC 4373 (placas planas de fibrocemento) y las propiedades físicas mecánicas son sometidas bajo norma ASTM. La Tabla 1 muestra las principales propiedades físico-mecánicas del material utilizado.

TABLA 1. PROPIEDADES FISICOMECÁNICAS DE FIBROCEMENTO SUPERBOARD [3]

\begin{tabular}{|c|c|c|c|}
\hline Propiedad & $\begin{array}{l}\text { Valor } \\
\text { Promedio }\end{array}$ & Unidad & Ensayo \\
\hline Absorción & 32.0 & $\%$ & ASTM C 1185 \\
\hline Densidad (seca al horno) & 1.25 & $\mathrm{~kg} / \mathrm{cm}^{3}$ & NTC 4373 \\
\hline Contenido de humedad & 10.0 & $\%$ & NTC 4373 \\
\hline Movimientos hídricos & & & \\
\hline $\begin{array}{l}\text { Estiramiento seco al aire saturado Encogimiento de saturado a seco al } \\
\text { horno }\end{array}$ & $\begin{array}{l}0.55 \\
2.39\end{array}$ & $\mathrm{~mm} / \mathrm{m}$ & $\begin{array}{l}\text { Redco } \\
\text { Procedure }\end{array}$ \\
\hline Movimientos térmicos & & & \\
\hline Encogimiento de $105^{\circ} \mathrm{C}$ a $25^{\circ} \mathrm{C}$ & $6,5 \times 10^{6}$ & $\mathrm{~m} / \mathrm{m}^{\circ} \mathrm{C}$ & $\begin{array}{l}\text { Redco } \\
\text { Procedure }\end{array}$ \\
\hline Seco longitudinal & 7,902 & & \\
\hline $\begin{array}{l}\text { Seco transversal } \\
\text { Saturado longitudinal }\end{array}$ & $\begin{array}{l}6,799 \\
5,769\end{array}$ & $\mathrm{MPa}$ & ASTM D1037 \\
\hline Saturado transversal & 4,738 & & \\
\hline Seco al ambiente longitudinal & 15.0 & & \\
\hline $\begin{array}{l}\text { Seco al ambiente transversal } \\
\text { Saturado longitudinal }\end{array}$ & $\begin{array}{l}9.0 \\
10.5\end{array}$ & $\mathrm{MPa}$ & NTC 4373 \\
\hline Saturado transversal & 6.5 & & \\
\hline Conductividad térmica & 0.26 & $\mathrm{~W} / \mathrm{m}^{\circ} \mathrm{K}$ & ASTM C518 \\
\hline Resistencia a la tracción del clavo En húmedo & 32.0 & & \\
\hline En seco & 64.7 & $\mathrm{~kg}$ & ASTM D1037 \\
\hline $\begin{array}{l}\text { Resistencia al impacto (Charpy) Seco al horno } \\
\text { Saturado }\end{array}$ & $\begin{array}{l}1.56 \\
2.86\end{array}$ & $\mathrm{KJ} / \mathrm{m} 2$ & ASTM D256 \\
\hline Resistencia el fuego & 0.0 & & \\
\hline Índice de expansión de la llama Índice de propagación de humo & 0.0 & & ASTM E84 \\
\hline
\end{tabular}

\subsection{Equipos y herramientas}

El equipo utilizado para el proceso de mecanizado es una fresadora CNC de 3 y 4 ejes, marca ROLAND, modelo MDX 40A. Este es un centro de mecanizado enfocado en la creación de prototipos 3D, para materiales como polímeros de ingeniería, maderas, resinas y materiales compuestos, para aplicaciones como: carcasas, arte y esculturas en 3D y grabado de placas (Figura ). Las especificaciones técnicas del equipo relevantes para el proceso de mecanizado se listan en la Tabla 2. Este centro de mecanizado orientado a la fabricación de prototipos por sustracción de material tiene la ventaja de ser abierto en su programación y permite trabajar a bajas velocidades de desplazamiento, característica que permite trabajar materiales duros como el fibrocemento. 


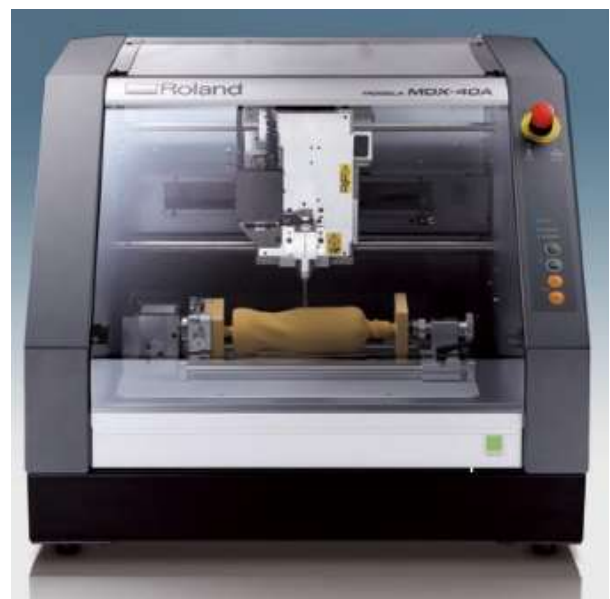

Figura 2. Fresadora ROLAND MDX 40ª (Imagen tomada de [11])

TABLA 2. ESPECIFICACIONES TÉCNICAS FRESADORA ROLAND MDX 40 A

\begin{tabular}{ll}
\hline Especificación & Valor \\
\hline Volumen de trabajo & $\mathrm{X}, \mathrm{Y}, \mathrm{Z}=305 \times 305 \times 105(\mathrm{~mm})$ \\
\hline Peso máximo del material a fabricar & $4(\mathrm{~kg})$ \\
\hline Sistema de Control ejes $\mathrm{X}, \mathrm{Y}, \mathrm{Z}$ & Motor Paso a Paso \\
\hline Velocidades de operación & $\mathrm{X}, \mathrm{Y}: 7$ a $3000(\mathrm{~mm} / \mathrm{min})$ \\
& $\mathrm{Z:} 7$ a $1800(\mathrm{~mm} / \mathrm{min})$ \\
\hline \multirow{2}{*}{ Resolución del Software } & $0.01 \mathrm{~mm} / \mathrm{Paso}(\mathrm{RML}-1)$ \\
\hline Resolución Mecánica & $0.001 \mathrm{~mm} /$ paso $(\mathrm{NC} \mathrm{code})$ \\
\hline Motor del Husillo & $0.002 \mathrm{~mm} /$ paso \\
\hline Velocidad del Husillo & Motor DC, sin escobillas \\
\hline
\end{tabular}

El proceso de mecanizado se realizó con dos herramientas de corte, una para el proceso de desbaste y otra para el proceso de acabado. Para el proceso de desbaste, se utilizó una fresa de carburo de tungsteno de 2 filos, Plana de 3/16" de diámetro, con una longitud total de 2" y longitud de flauta de 3/8" (Figura 3a). Para el proceso de acabado, se utilizó una Fresa de carburo de tungsteno de 2 filos, redonda de 3/16" de diámetro, con longitud total de 2" y longitud de flauta de 3/8" (Figura 3b). El carburo de tungsteno, material de las fresas con las que se realizaron los procesos de mecanizado, es una aleación de Cerámica-metal, dura, con gran resistencia al desgaste, a la compresión y a la temperatura, por ello su principal aplicación es la elaboración de herramientas de corte, maquinaria industrial y abrasivos [12].

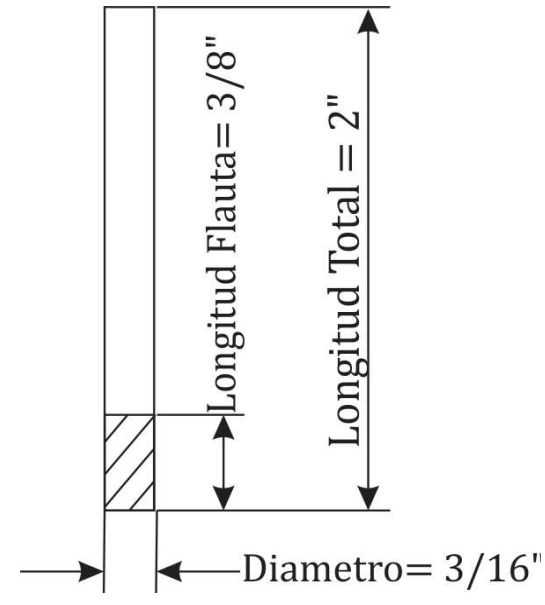

a)

Figura 3. a) Herramienta proceso desbaste. b) Herramienta proceso acabado 


\subsection{Software}

Para la parametrización en el software CAD de la superficie a maquinar en la placa, se utilizó el software Rhinoceros 5 con el plug-in o complemento Grasshopper en su versión 0.9.0076. Al ser un software CAD de forma libre, para la parametrización de modelos es necesario el uso Grasshopper, para la edición de algoritmos generativos de forma gráfica para la programación y parametrización de los modelos a diseñar [13].

Rhinoceros, es un software de modelado 3D de forma libre, utilizado como herramienta de diseño e ingeniería para la concepción, análisis y fabricación de prototipos y productos sin importar su complejidad, gracias a que permite trabajar con superficies, solidos, nubes de puntos y mallas poligonales de forma precisa, intuitiva y rápida. [14] Se utilizó RhinoCAM para la generación de los códigos $G$ que permitieron la ejecución de los diferentes procesos de mecanizado en la fresadora ROLAND MDX 40; este último es un software de manufactura asistido por computador (CAM) que permite la programación de máquinas de control numérico computarizado (CNC) en 2.5, 3, 4 y 5 ejes, permitiendo la simulación de procesos para la fabricación de prototipos, moldes, herramientas, etc. [15].

\subsection{Parametrización de la superficie en software CAD}

Se trabajó un módulo definido por una superficie tridimensional de doble curvatura para la evaluación del material a ser mecanizado. Este módulo fue parametrizado en un patrón rectangular que varía según la cantidad de veces que se desea replicar la superficie a lo ancho y a lo largo de la placa a mecanizar. Este módulo adapta sus dimensiones según la placa, definidas por su ancho, largo y profundidad. El módulo principal para trabajar fue un paraboloide hiperbólico (ver Figura ). Esta superficie tridimensional en ocasiones es conocida como silla de montar por su semejanza a esta [16].

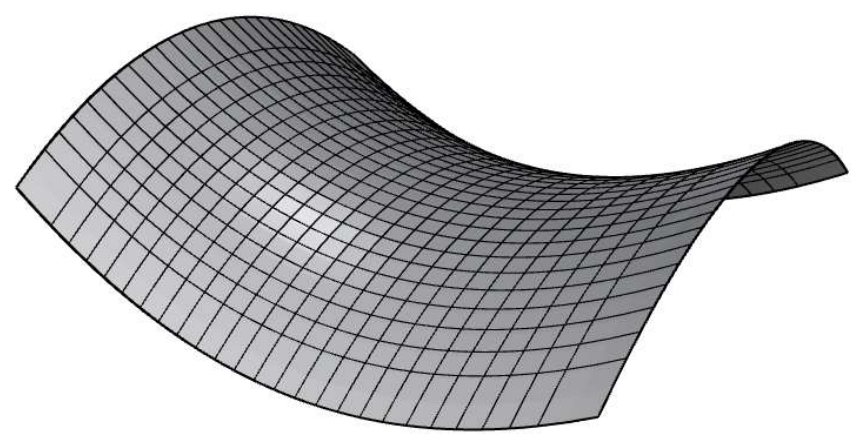

Figura 4. Gráfico paraboloide hiperbólico

Esta superficie es definida por la ecuación $" \frac{x^{2}}{a^{2}}-\frac{y^{2}}{b^{2}}=\frac{z}{c} \quad$ (1)” en su forma Canónica.

$$
\frac{x^{2}}{a^{2}}-\frac{y^{2}}{b^{2}}=\frac{z}{c}
$$

Donde $a, b$ y $c$ son números reales diferentes a 0 y definen un valor de escala (positivo o negativo) correspondiente a los ejes coordenados $x, y, z$. Para graficar la superficie en el software CAD, se empleó la ecuación $\frac{x^{2}}{a^{2}}-\frac{y^{2}}{b^{2}}=\frac{z}{c} \quad$ (1) con sus respectivas ecuaciones paramétricas (2) a (4).

$$
\begin{gathered}
x=a \cdot u \\
\mathrm{y}=\mathrm{b} . v \\
\mathrm{z}=\left(u^{2}-v^{2}\right) * \mathrm{c}
\end{gathered}
$$

Donde $u, v, c$ son los valores por evaluar con; $-\infty\rangle u>\infty,-\infty<v>\infty$ y $-\infty<c>\infty$.

Como modelo de cálculo para la generación de la geometría de la placa en el software CAD, se asumieron valores de entrada del usuario y valores de cálculo o de salida. Los valores de entrada del usuario, son los datos numéricos que el usuario ingresa en el modelo de cálculo para la generación de la geometría de la placa, según la necesidad de las dimensiones de ancho, largo y profundidad de la placa y el número de veces que desea replicar el módulo (Figura) en los ejes $x$ e $y$. La Tabla 3 especifica los valores de ingreso por parte del usuario 
Una alternativa para la producción de superficies de doble curvatura en láminas de fibrocemento a través de mecanizado de

para el modelo de cálculo, y la nomenclatura asociada a cada una de las variables necesarias y que se emplean en las ecuaciones subsiguientes.

TABLA 3. VALORES DE ENTRADA DEL USUARIO

\begin{tabular}{lll}
\hline Especificación variable & Designación & Especificación de la variable \\
\hline Largo placa & $L p$ & Valor del largo de la placa a lo largo del eje $y$. \\
\hline Ancho placa & $A p$ & Valor del ancho de la placa a lo largo del eje $x$. \\
\hline Profundidad placa & $P p$ & Valor de el espesor total de la placa. \\
\hline Numero de módulos a lo largo de la placa & $N s l$ & Valor de Numero de módulos a replicar a lo largo del eje $y$, \\
\hline Numero de módulos a lo ancho placa & $N s a$ & Valor de Numero de módulos a replicar a lo largo del eje $x$, \\
\hline
\end{tabular}

Para determinar el largo y ancho total del módulo a replicar en la placa, se calculó a través de la división del número de veces que se debe replicar este a lo largo y ancho de la placa. Para el largo del módulo, el valor máximo está dado por:

$$
y_{M}=L p / N s l
$$

Para el ancho máximo del módulo, el valor está dado por:

$$
x_{m}=A p / N s a
$$

De forma gráfica, el modelo de generación de los módulos y la designación de variables se representa en la Figura 5.

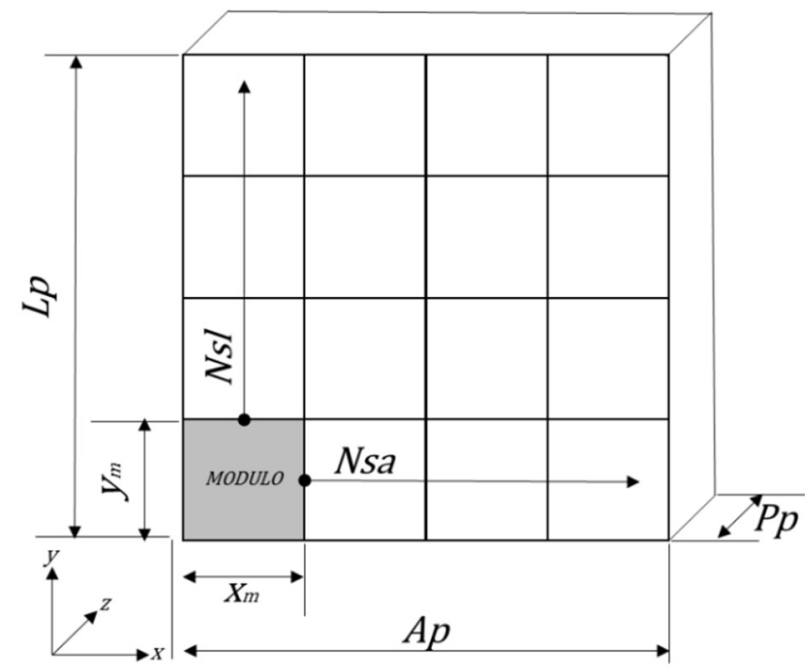

Figura 5. Representación modelo generación superficie en placa.

Para calcular la profundidad máxima del módulo en la placa, se estableció este como un valor constante, en donde esta profundidad es:

$$
Z_{M}=P_{p} / 1.25
$$

El valor de 1.25 en la ecuación $\boldsymbol{Z}_{\boldsymbol{M}}=\boldsymbol{P}_{\boldsymbol{p}} 1.25$ (7), tiene el fin de calcular un $25 \%$ del espesor de la placa y permitir generar un espesor de material sin mecanizar, y garantizar así la unión entre los diferentes módulos que conformarán un solo volumen. De forma gráfica el modelo de generación de los módulos en función de la profundidad se representa en la Figura 6. 


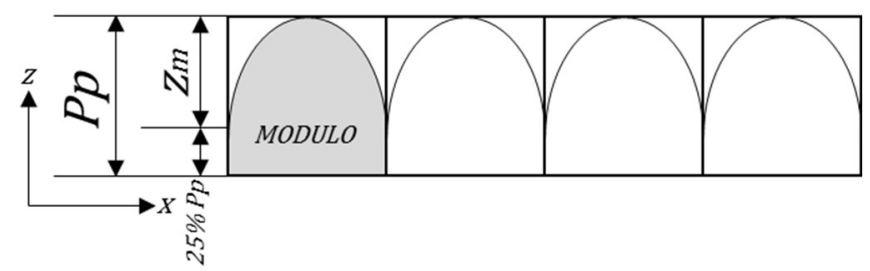

Figura 6. Representación modelo de generación de la superficie en función de la profundidad de la placa.

TABLA 4. VALORES DE ENTRADA DE USUARIO DE GEOMETRÍA PARA PROCESO DE MAQUINADO.

\begin{tabular}{lll}
\hline Designación & Valor & Unidades \\
\hline$L p$ & 150 & $\mathrm{~mm}$ \\
\hline$A p$ & 150 & $\mathrm{~mm}$ \\
\hline$P p$ & 10 & $\mathrm{~mm}$ \\
\hline $\mathrm{Nsl}$ & 8 & unidades \\
\hline $\mathrm{Nsa}$ & 8 & unidades \\
\hline
\end{tabular}

Los valores de entrada de usuario con los que se generó la geometría para el proceso de mecanizado se especifican en la TABLA 4. Así, para los valores asignados en la TABLA 4. los valores del tamaño del módulo

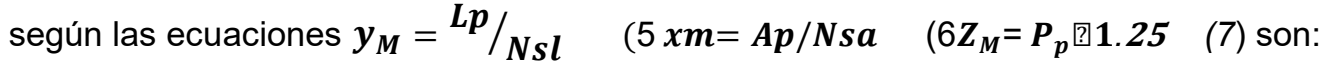

$$
\begin{gathered}
X_{m}=\frac{150 \mathrm{~mm}}{8}=18.75 \mathrm{~mm} \\
Y_{m}=\frac{150 \mathrm{~mm}}{8}=18.75 \mathrm{~mm} \\
Z_{m}=\left(\frac{10 \mathrm{~mm}}{1.25}\right)=8 \mathrm{~mm}
\end{gathered}
$$

El centro del módulo es ubicado en el punto del espacio $(x, y, z)$ en $(0,0,0)$, como se muestra en la Figura 7.

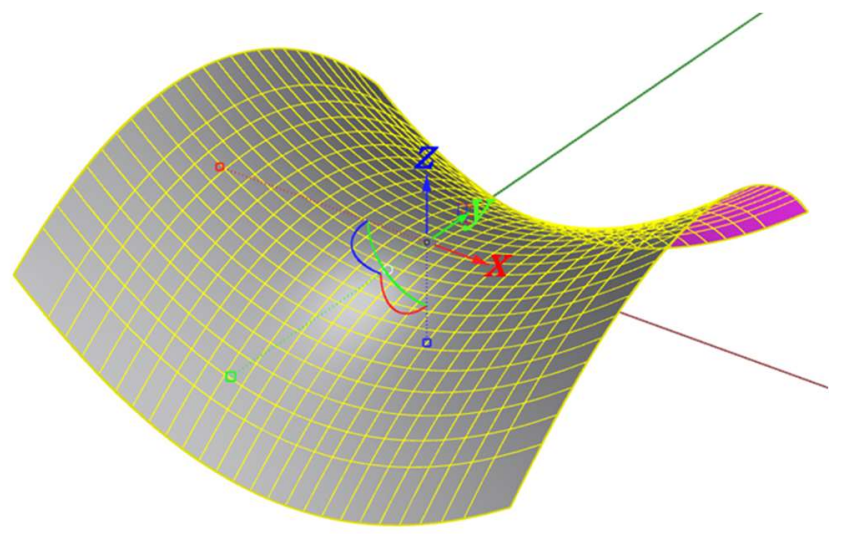

Figura 7. Origen módulo

Así los valores de $a, b, c$ en las ecuaciones $x=a . u$ se representan en la Figura 8, se definen como:

(2), $\mathrm{y}=\mathrm{b} \cdot v$

$(3) \mathrm{z}=\left(u^{2}-v^{2}\right) * \mathrm{c}$

(4), y que

$$
\begin{aligned}
& a=\frac{x_{m}}{2}=9.375 \mathrm{~mm} \\
& b=\frac{y_{m}}{2}=9.375 \mathrm{~mm}
\end{aligned}
$$


Una alternativa para la producción de superficies de doble curvatura en láminas de fibrocemento a través de mecanizado de

$$
c=\frac{z_{m}}{2}=4 m m
$$

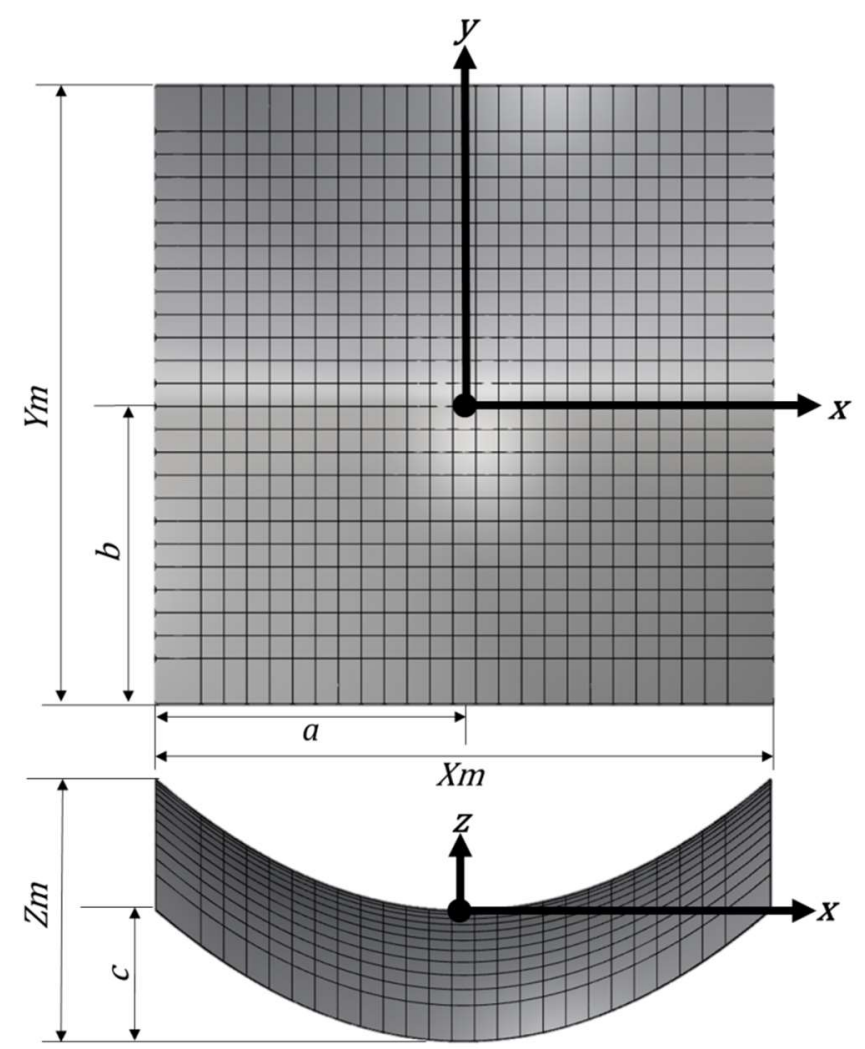

Figura 8. Representación gráfica de los valores $a, b, c$ del módulo

Para graficar la superficie del módulo individual, se asignó un rango de; $-1<u<1,-1<v<1$ y $c=4$, con el fin de delimitar las dimensiones en $X_{m}, Y_{m}, Z_{m}$ del módulo asignadas a la hora de realizar el trazado de este. Se determinó un número de 27 iteraciones en el rango asignado para el cálculo de los puntos del módulo en

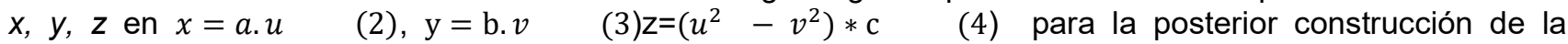
superficie por el software CAD. Al reemplazar los valores asignados en las ecuaciones $x=a . u \quad(2), \mathrm{y}=$ b. $v \quad(3) z=\left(\begin{array}{lll}u^{2}-v^{2}\end{array}\right) * c \quad(4)$, con las condiciones de rango e iteraciones asignadas, se generó una nube de puntos con 783 valores.

Luego de definida la nube de puntos en las coordenadas $x, y, z$, se generó la superficie del módulo a partir de la herramienta; "superficie a partir de nube de puntos" de Rhino. La cual se duplicó por medio de un patrón rectangular según los valores asignados en $\mathrm{Nsl}$ y $\mathrm{Nsa}$, con un valor de 8 para ambos casos. Para generar el resto del volumen se definió un conjunto de operaciones para construir una Polisuperficie cerrada a ser trabajada en RhinoCAM. La Figura 9 muestra el resultado del conjunto de operaciones realizadas en Grasshopper para la generación del volumen; cabe notar que este desarrollo está disponible online y se comparte el programa realizado en el foro oficial de las aplicaciones libre de Grasshopper 3D [17].

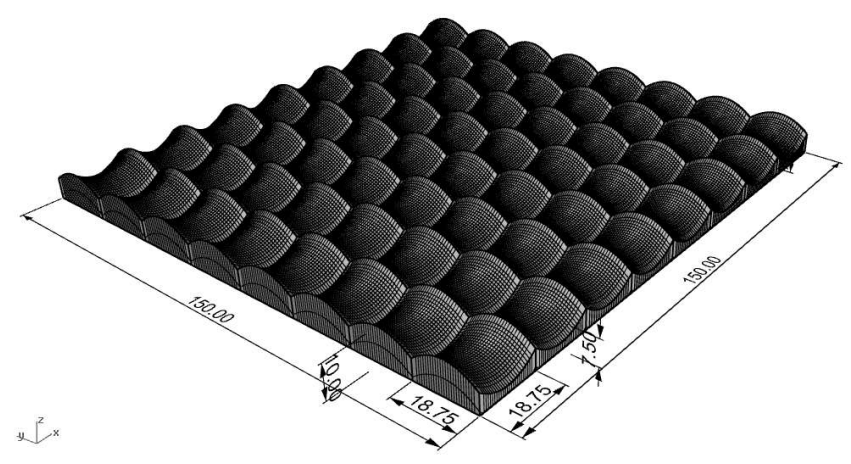


Figura 9. Geometría generada en Grasshopper para proceso de mecanizado.

\subsection{Generación de programa CAM para el maquinado de la Pieza y característica del proceso}

Para el proceso de mecanizado realizado en una lámina estándar lisa de 10mm de espesor, marca Superboard, se generaron 2 Códigos $\mathrm{G}$ o programas en Grashopper: uno para el proceso de desbaste y otro para el proceso de acabado. El proceso de desbaste es el primer paso que se realizó para retirar la mayor cantidad del volumen del material de la placa inicial de forma rápida y sin comprometer la geometría de la superficie. Este proceso se hace de forma escalonada y deja un margen en la periferia de la geometría [18] para posteriormente realizar el proceso de acabado. La Figura 10 muestra el proceso de desbaste realizados a la superficie generada por el software CAD.

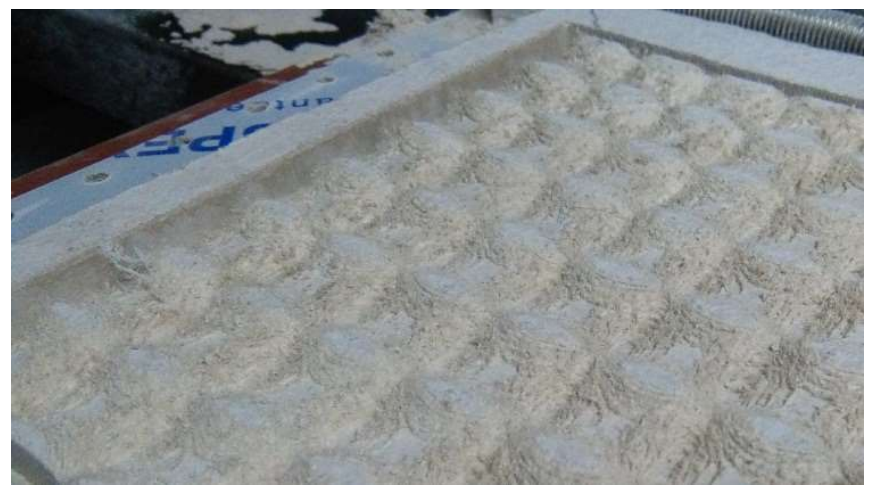

Figura 10. Proceso de desbaste de la lámina de fibrocemento

TABLA 5. PARÁMETROS CAM PARA EL PROCESO DE DESBASTE DE LA PLACA

\begin{tabular}{lll}
\hline Especificación & Valor & Unidades \\
\hline Velocidad del Usillo & 15.000 & $\mathrm{rpm}$ \\
\hline Profundidad de corte & 0.5 & $\mathrm{~mm}$ \\
\hline Velocidad de avance de corte & 180 & $\mathrm{~mm} / \mathrm{min}$ \\
\hline Stock o Margen diferencial con respecto a la geometría. final & 0.6 & $\mathrm{~mm}$ \\
\hline Dirección de avance de herramienta & Lineal mixta en dirección x & No aplica \\
\hline Paso de avance de herramienta & 0.5 & $\mathrm{~mm}$ \\
\hline Tiempo del proceso & 13 & $\mathrm{hr}$ \\
\hline
\end{tabular}

La Tabla 5 especifica los parámetros representativos introducidos en RhinoCAM correspondientes al proceso desbaste de la placa, con un proceso de "Horizontal Roughing". Este proceso fue simulado en el software para verificar y detectar fallas durante el proceso y así garantizar la correcta operación (Figura 11a). Los colores rojos representan los desplazamientos en vacío, los amarillos las entradas y salidas de la herramienta, y las líneas de color Cian indican los desplazamientos de sustracción de material.

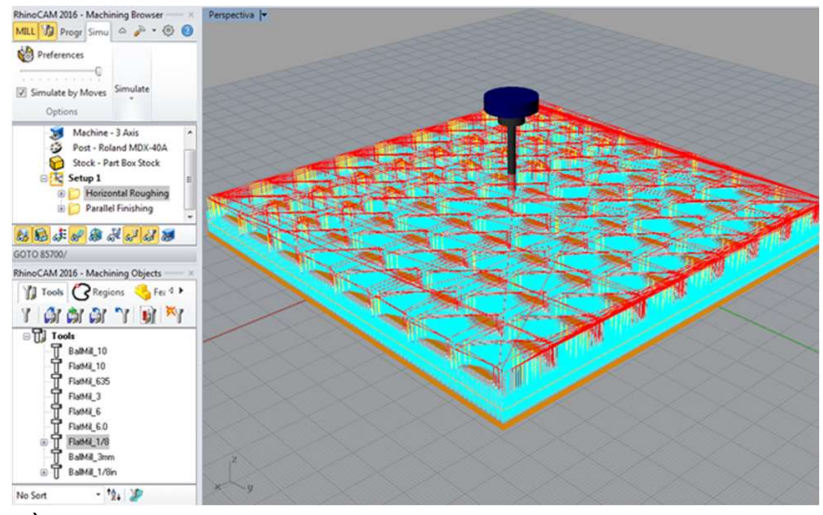

a)

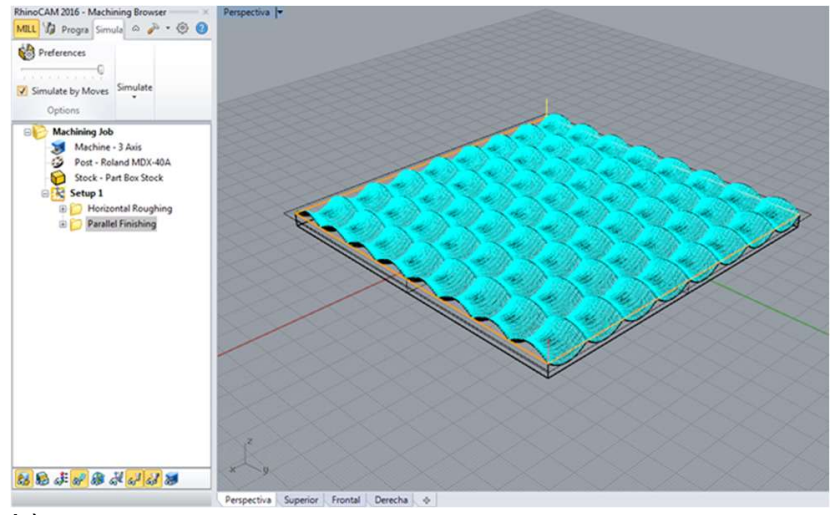

b)

Figura 21. a) Simulación CAM del proceso desbaste. b) Simulación CAM proceso acabado 
El proceso de acabado se realizó posterior al proceso de desbaste; con este se buscó obtener el mejor acabado superficial posible en el material, en el cual la superficie fuera lisa, sin marcas de herramientas, rayones o desprendimientos de material. Así como se realizó con el proceso de desbaste, el proceso de acabado es inicialmente simulado en software (Figura 11b), con los valores presentados en la Tabla 6, la cual especifica los parámetros representativos introducidos en RhinoCAM, correspondientes al acabado de la placa con un proceso de "Parallel Finishing". La Figura 12 muestra el resultado final del proceso de acabado realizado a la superficie generada por el software CAD.

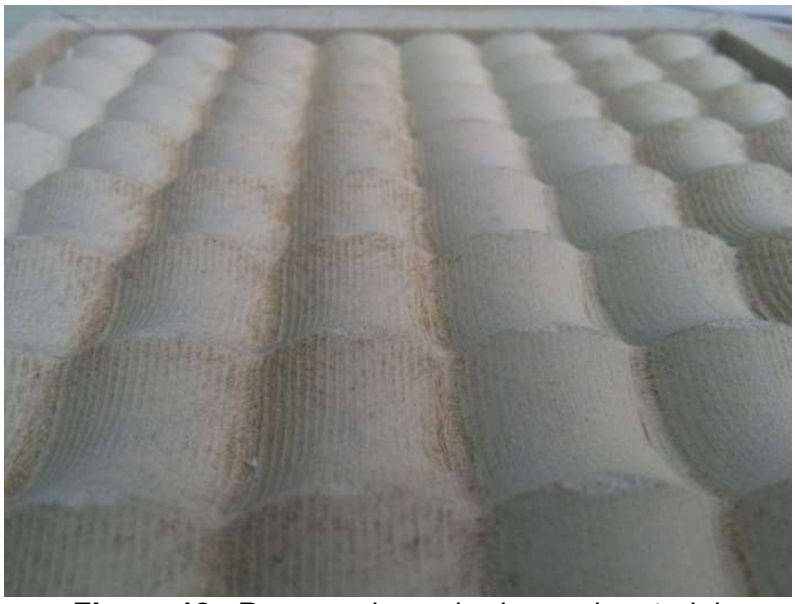

Figura 12. Proceso de acabado en el material

TABLA 6. PARÁMETROS CAM PARA EL PROCESO DE ACABADO DE PLACA

\begin{tabular}{lll}
\hline Especificación & Valor & Unidades \\
\hline Velocidad de la herramienta & 15.000 & $\mathrm{rpm}$ \\
\hline Profundidad de corte & 0.6 & $\mathrm{~mm}$ \\
\hline Velocidad de avance de corte & 180 & $\mathrm{~mm} / \mathrm{min}$ \\
\hline Stock o Margen diferencial con respecto a la geometría. final & 0.03 & $\mathrm{~mm}$ \\
\hline Dirección de avance de herramienta & Lineal mixta en dirección x & No aplica \\
\hline Tiempo del proceso & 4.65 & $\mathrm{hr}$ \\
\hline
\end{tabular}

\section{DISCUSIÓN DE LOS RESULTADOS}

Al obtener los códigos $\mathrm{G}$ de RhinoCAM, se realizó el montaje y ejecución de los procesos para el mecanizado de la superficie en la placa (ver Figura 3).

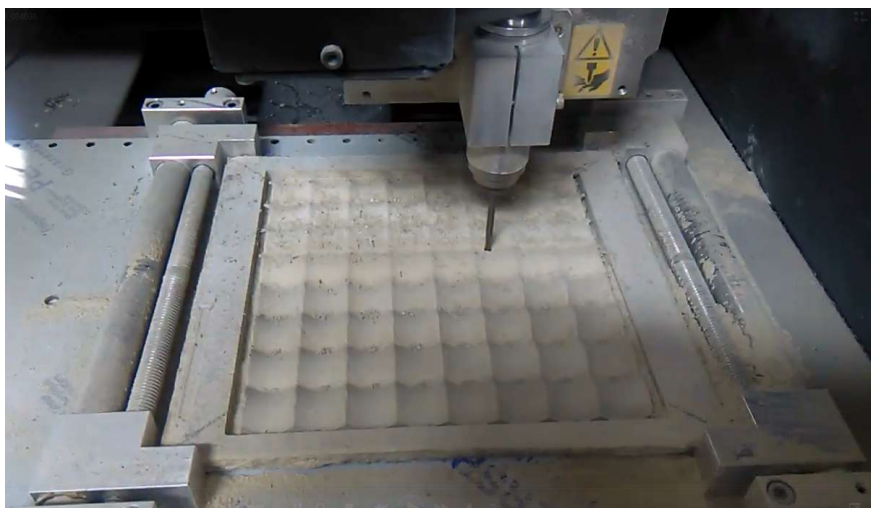

Figura 3. Montaje y proceso mecanizado

Como resultado final se obtuvo la placa parametrizada en el software CAD (ver Figura 4) 


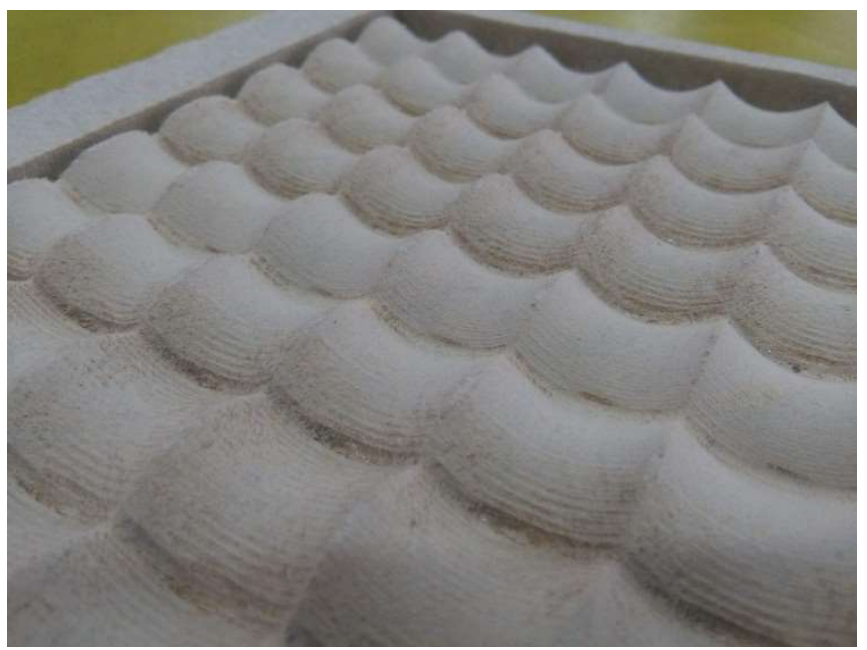

Figura 4. Resultado final placa mecanizada

El acabado obtenido durante el proceso realizado es aceptable, aunque se presentan en él marcas del paso de la herramienta en su superficie (Figura 4). Para mejorar esto es necesario realizar otro proceso de acabado bajo los mismos parámetros especificados en Tabla 6 pero cambiando la dirección de avance de herramienta a lineal mixta en la dirección $Y$ con el fin de disminuir dichas marcas.

Para ampliar la cantidad de piezas a maquinar por medio de esta técnica de manufactura, debido a la restricción de los espesores comerciales disponibles de láminas de fibrocemento, estas se deban aglomerar entre sí para obtener mayores espesores, haciendo uso de adhesivos con base de Poliuretano (PU) o epóxicas de altas prestaciones mecánicas.

El mecanizado de superficies de doble curvatura en fibrocemento puede ser aplicado en la fabricación de piezas de diferentes sectores industriales. El procedimiento descrito en este artículo sirve como referente para la fabricación de piezas y productos que trasciendan los usos tradicionales que se han dado al fibrocemento, ya que posibilita la aplicación en nuevos contextos dada las propiedades del material. Se puede pensar en el fibrocemento como un material con utilidad más allá del cerramiento de espacios arquitectónicos y de la construcción; en la Tabla 7 se proponen diferentes aplicaciones en diferentes tipos de industrias. Estas aplicaciones deben ser evaluadas según su relación costo-beneficio, cantidad de unidades a producir, normatividad, prestaciones mecánicas, eléctricas y químicas y desempeño, que realmente hagan de la aplicación de este material una alternativa viable para la industria comparándolo con el uso de materiales tradicionales.

\subsection{Trabajos futuros}

La obtención de superficies de doble curvatura en fibrocemento permite diseñar y fabricar piezas con nuevas aplicaciones y geometrías de mayor complejidad, que pueden ir más allá de la aplicación actual que se le da a este material en el sector de la construcción. La exploración de aplicaciones en el sector de ingeniería, como por ejemplo aplicaciones de aislantes eléctricos y térmicos, diseño de moldes para el vaciado de resinas y materiales compuestos o piezas que requieran de aceptables prestaciones mecánicas que deben estar expuestas a condiciones de intemperie, son una alternativa para explorar en el futuro.

Este material, en los sectores de diseño y arquitectura, la conceptualización de mobiliario, artefactos, elementos decorativos, elementos arquitectónicos, etc. en los contextos; público, doméstico e institucional, puede ser una alternativa diferenciadora a lo que tradicionalmente se realiza. Con nuevos tipos de ensamble, acabados, texturas y geometrías.

Por otro lado, es importante notar que el proceso descrito se ejecutó bajo limitaciones técnicas en equipos y herramientas de restringen la aplicación del proceso a escala industrial. Por ello, la optimización del proceso de mecanizado descrito a nivel industrial es un camino aun por recorrer, donde se hace necesario que disminuyan los tiempos de proceso, se mejore el acabado superficial y el desgaste de la herramienta para aumentar el costobeneficio del maquinado de este tipo de material y sea más atractivo para la industria. 
Una alternativa para la producción de superficies de doble curvatura en láminas de fibrocemento a través de mecanizado de

An alternative to produce doubly curved surfaces in fiber cement sheets using three-axe machining

TABLA 7. POSIBLES APLICACIONES PARA EL MAQUINADO DE SUPERFICIES EN FIBROCEMENTO EN LA INDUSTRIA.

\begin{tabular}{ll}
\hline Sector o Industria & Aplicación \\
\hline & Moldes para el vaciado de resina o el laminado de materiales compuestos. \\
& $\begin{array}{l}\text { Piezas mecánicas o cerramientos sometidos a altas temperaturas como; hornos, } \\
\text { dispositivos de sujeción y contenedores de material. }\end{array}$ \\
$\begin{array}{l}\text { Ingeniería y } \\
\text { manufactura }\end{array}$ & Aislantes térmicos de sistemas de refrigeración o recubrimientos. \\
& Aislantes acústicos como; cerramientos insonorizados o cajas de sonido. \\
& $\begin{array}{l}\text { Prototipado rápido como sustituto de la madera para piezas con mejores prestaciones } \\
\text { mecánicas y estéticas. }\end{array}$ \\
\hline Eléctrico & Aisladores eléctricos. \\
Construcción y & Señalización institucional \\
arquitectura & Detalles arquitectónicos como; listones, fachadas o acabados. \\
\hline Arte y diseño & Elementos cortafuegos como; puertas, ventanas y división de espacios. \\
\hline & Mobiliario público, domestico, comercial e institucional, como; mesas, sillas, sistemas de \\
& almacenamiento, y detalles de diseño. \\
Mobiliario & Uniones de ensamble como; soportes y esquineros. \\
& Desarrollo de espacios efímeros como estand comerciales y publicitarios \\
& Contenedores especiales de residuos orgánicos, químicos y sólidos.
\end{tabular}

\section{CONCLUSIONES}

El mecanizado de superficies de doble curvatura en fibrocemento es viable de realizar en piezas de baja producción o de aplicaciones especiales bajo los parámetros realizados en este trabajo, donde el equipo utilizado no es de uso industrial y las herramientas empleadas no son especializadas para el mecanizado de materiales compuestos de mayor dureza y abrasión. Sin embargo, es precisamente esta limitación lo que hace que el tiempo de maquinado y el desgaste de las herramientas no hacen atractivo este procedimiento para uso industrial bajo estas condiciones, restringiendo la adopción de este método para altos volúmenes de producción.

Para mejorar estos aspectos del tiempo de mecanizado y desgaste de las herramientas, se deben emplear equipos de uso industrial que soporten mejor las condiciones mecánicas que exige el material, y que tengan incorporado un sistema de refrigeración que permita trabajar los procesos de mecanizado a mayor velocidad, protegiendo la vida útil de la herramienta.

Este proceso tiene potencial de ser utilizado en aplicaciones de prototipado y diseño de piezas especiales que requiera de propiedades térmicas, aislantes y de resistencia mecánica y química que el material posee. Por otro lado, mejorando el proceso en tiempo y desgaste de herramienta, este puede ser aplicado a nivel industrial y arquitectónico., en procesos tales como el diseño de mobiliario, aislantes eléctricos y aplicaciones arquitectónicas especiales.

\section{AGRADECIMIENTOS}

Los autores agradecen por el apoyo técnico y procedimental a:

- $\quad$ Ing José Ovidio Cardona, director Rhinofab Estudio, Universidad Pontificia Bolivariana.

- Ing Jorge Eladio Torres Gómez, Instructor Centro para el Desarrollo del Hábitat y la Construcción; Servicio Nacional de Aprendizaje SENA.

- Ing Nelson Castaño Ciro, instructor Investigador SENNOVA, Centro para el Desarrollo del Hábitat y la Construcción; Servicio Nacional de Aprendizaje SENA. 


\section{REFERENCIAS}

[1] J. Rodríguez, J. Tobón, M. Frías y M. I. Sánchez de Rojas, «Aprovechamiento de un residuo del carbón para reducción del impacto ambiental de la minería del carbón en Colombia: estudio del potencial de uso en la industria del cemento,» Revista Cintex, vol. 23, n² 2, pp. 95-102, 2018.

[2] Eternit, «www.eternit.com.co,» 01 2018. [En línea]. Available: https://www.eternit.com.co/documents/32456/170883/CARTILLA-ETERBOARD.pdf/0c954230-0837-4087-ab9aec411cb96ecd. [Último acceso: 0807 2019].

[3] N. Montes Valencia, «Cementos Alcalinos: Materiales de Construcción Ecológicos,» Revista Cintex, vol. 19, pp. 109-125, 2014.

[4] E. Mejía, L. Osorno y J. Ospina, «Microorganismos Hierro-Azufre Oxidantes Una Alternativa Biotecnológica,» Revista Cintex, vol. 19, pp. 63-77, 2014.

[5] J. Rodríguez, «Aprovechamiento de un residuo del carbón para reducción del impacto ambiental de la minería del carbón en Colombia: estudio del potencial de uso en la industria del cemento,» CINTEX, vol. 23, pp. 95-102, 2018.

[6] C. M. Tamayo Domínguez, "Caracterización de la administración de la tecnología de máquinas herramientas C.N.C instaladas en el Valle de Aburrá,» Revista CINTEX, vol. 13, pp. 20-26, 2008.

[7] L. M. García, «Estudio del mecanizado de materiales compuestos,» 06 2010. [En línea]. Available: https://www.kimerius.com/app/download/5781437488/Estudio\%20del\%20mecanizado\%20de\%20materiales $\% 20$ compuestos.pdf.

[8] F. Hoyos Gómez, J. D. Betancur Gómez, D. Osorio Patiño y J. G. Ardila Marín, «Construcción de curvas de factor de concentración de esfuerzos por medio de simulaciones,» Revista Cintex, vol. 21, n 1, pp. 35-43, 2016.

[9] J. A. Patiño Murillo, J. J. Castro-Maldonado, Y. C. Gutiérrez-Sandoval, J. I. Leal-Santafé y O. HurtadoFigueroa, «Estudio del comportamiento de muestras de mortero natural sometidas a esfuerzo de compresión,» Lámpsakos, $n^{\circ} 20$, pp. 22-28, 2018.

[10] M. Ardanuy, "Cellulosic fiber reinforced cement-based composites: A review of recentresearch,» Construction and Building Materials, vol. 79, pp. 115-128, 2015.

[11] Roland DG corporation, «Roland,» 2019. [En línea]. Available: http://support.rolanddga.com/Docs/Documents/departments/Technical\%20Services/Manuals\%20and\%20Guides /MDX-40A_USE_EN_R1.pdf. [Último acceso: 1107 2019].

[12] K. Liu, «CBN tool wear in ductile cutting of tungsten carbide,» de Wear of materials, Washington, 2003.

[13] Scott Davidson, «grasshopper,» Scott Davidson, 2019. [En línea]. Available: https://www.grasshopper3d.com/. [Último acceso: 0908 2019].

[14] Robert McNeel \& Associates, «Rhinoceros,» Robert McNeel \& Associates, 2019. [En línea]. Available: https://www.rhino3d.com/6/features. [Último acceso: 0908 2019].

[15] MecSoft Corporation, «MecSoft Corporation,» 2019. [En línea]. Available: https://mecsoft.com/DataSheets/RhinoCAM2019/DataSheet.pdf. [Último acceso: 1508 2019].

[16] J. STEWART, CAlculo de Varias Variables, México: Cengae Learning, 2012.

[17] R. Isaza Escobar, «Parametrizacion sllla de montar,» 0609 2019. [En línea]. Available: https://www.food4rhino.com/resource/parametrizacion-silla-de-montar.

[18] O. E. Guerrero, Procesos de manufactura en ingenieria Industrial, Bogota: Universidad Nacional Abierta y a Distancia , 008.

[19] B. V. Acoleyen, «HATSCHEK PROCESS». Nivel Mundial Patente WO 2017/001230 A2, 5012017.

[20] J. E. Mejia-Ballesteros, "Effect of mineral additions on the microstructure and properties of blended,» Cement and Concrete Composites, vol. 98, pp. 46-60, 2019.

[21] Y. Yang, "Uniaxial compression mechanical properties and fracture characteristics of,» Composite Structures, vol. 212, pp. 148-158, 2019.

[22] A.M.Cooke, "Durability of Autoclaved Cellulose Fiber Cement Composites,» Building Materials and Technology, vol. 7, pp. 2-37, 2000.

[23] COLOMBIT, "COLOMBIT,» 2018. [En Available: https://www.etex.com.co/productos/superboard/estandar.html?marca=122. [Último acceso: 2907 2019]. 